\title{
Impact of the Nutritional Risk Score in Fast-Track Colon Surgery
}

\author{
M. Hübner S. Müller M.Schäfer P.A. Clavien N. Demartines \\ Department of Visceral and Transplantation Surgery, University Hospital Zurich, Zurich, Switzerland
}

\section{Key Words}

Malnutrition $\cdot$ Colon surgery $\cdot$ Fast-track $\cdot$ Complications

\begin{abstract}
Background: Patients at nutritional risk reveal an increased morbidity. Fast-track (FT) programs in colonic surgery have shown reduced complications and hospital stay. We aimed to assess the effect of FT programs on patients at nutritional risk. Methods: In a randomized trial (NCT00556790), we compared complications after open colonic surgery with either a FT program or standard care (SC). A subgroup analysis was performed in 67 patients for whom a prospective nutritional risk score (NRS) was available. Results: The SC and FT groups did not differ regarding patient characteristics or prevalence of NRS $\geq 3$ (SC 8/31, FT 7/36, p = 0.569). Patients with SC had more complications $(14 / 31$ vs. $8 / 36, p=0.044)$ and a longer hospital stay ( 9 vs. 5 days, $\mathrm{p}<0.0001$ ). No major complication occurred in patients with an NRS $<3$. Patients at nutritional risk had a high complication rate regardless of SC or FT ( $6 / 8$ and $5 / 7$, respectively, $p=1.000$ ). Median hospital stay was shorter in FT (7 (range 5-30) days) versus SC patients (14.5 (range 8-30) days, $\mathrm{p}=0.164$ ). Conclusions: Patients with a NRS $\geq 3$ have an increased postoperative morbidity even within a FT program. They should be identified by nutritional screening and might benefit from nutritional supplements.

Copyright $\odot 2010$ S. Karger AG, Basel
\end{abstract}

\section{Introduction}

Preoperative malnutrition is a well-known risk factor for postoperative complications, and up to $40 \%$ of patients undergoing major abdominal surgery are at nutritional risk [1-6]. Increased postoperative morbidity represents the main cause of prolonged hospital stay, longer recovery time, and increased use of health care resources. Considerable efforts have been made during recent years to overcome this vicious circle [7-9]. Patient screening for malnutrition and early nutritional support, as well as fasttrack (FT) programs have provided beneficial effects [710]. Both nutritional support and FT programs primarily aim to enhance recovery and to decrease postoperative morbidity by minimizing perioperative organ dysfunction. Secondary beneficial effects are the shortened hospital stay and decreased costs [4, 5, 7-10].

Existing FT protocols do not include preoperative nutritional screening or even exclude patients at nutritional risk $[9,10]$. So far, no published series has assessed the effects of FT programs on this frail subset of patients. It remains to be elucidated whether these patients have the same benefits from enhanced recovery programs, e.g. reduced complications and hospital stay, or if they experi-

Preliminary data were presented as late-breaking abstract (LB11) at the 29th ESPEN Congress, Prague, September 2007, and at the 95th Annual Meeting of the Swiss Society of Surgery, Basel, May 2008.

\section{KARGER}

Fax +4161306 1234 E-Mail karger@karger.ch www.karger.com
Martin Hübner, MD

Department of Visceral Surgery

University Hospital Lausanne, Rue du Bugnon 46

$\mathrm{CH}-1011$ Lausanne (Switzerland)

Tel. +41 2131424 18,Fax +41 2131424 11, E-Mail martin.hubner@ chuv.ch 
Table 1. Screening for nutritional risk - the nutritional risk score

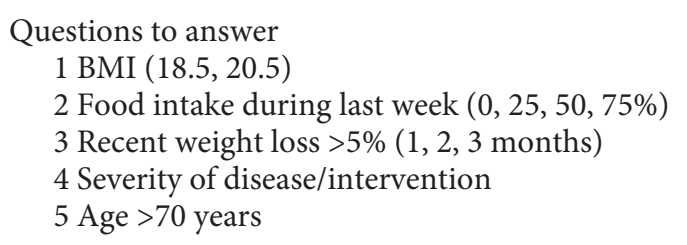

\begin{tabular}{llll}
\hline & $\begin{array}{l}\text { Mild } \\
\text { score 1 }\end{array}$ & $\begin{array}{l}\text { Moderate } \\
\text { score 2 }\end{array}$ & $\begin{array}{l}\text { Severe } \\
\text { score 3 }\end{array}$ \\
\hline $\begin{array}{l}\text { Nutritional status } \\
\text { BMI }\end{array}$ & & $18.5-20.5$ & $<18.5$ \\
$\quad$ Food intake, $\%$ & $50-75$ & $25-50$ & $<25$ \\
$\quad$ Weight loss $>5 \%$ & 3 months & 2 months & 1 month \\
Disease severity example & Hip fracture & Major surgery & BM transplant \\
Age, years & $>70$ & & \\
\hline
\end{tabular}

Nutritional risk assessment adapted from Kondrup et al. [1, 2]. The nutritional status was evaluated by calculation of the body mass index (BMI), estimation of the actual food intake in the preceding week (\% of normal requirements), and by recent weight loss (questions 1-3). The respective scores are attributed according to the answers to the questions. Only the highest score of the three nutritional items enters the final score. The severity of dis- ease/intervention is graded accordingly; example lists available in $[1,2]$. One score is added for patients older than 70 years (adjustment for frailty of elderly). The composite score of the highest scores of the three categories gives values between 0 and 7. Individuals with a NRS $\geq 3$ are at risk of increased morbidity and are likely to benefit from nutritional support $[1,2]$. ence no beneficial or even devastating effects due to the more aggressive postoperative care. Since we recently published a prospective randomized trial on FT in patients undergoing open colon resection [8], we had the unique opportunity to analyze the outcome of the subgroup of patients at increased nutritional risk receiving a FT regimen compared to conventional standard care (SC).

The aim of the current study was to assess the impact of the FT program versus SC on patients stratified by their nutritional risk, with particular focus on patients at increased nutritional risk.

\section{Patients and Methods}

A prospective randomized trial on FT versus SC was performed in 156 patients undergoing open elective colon resection at four surgical departments in Switzerland (NCT00556790) [8]. Preoperative nutritional assessment was systematically performed by using the nutritional risk score (NRS) in two of the four centers, hence the present study includes 67 patients for final analysis.

The NRS integrates the patients' nutritional status, the severity of the disease or intervention, and age in a multimodal screening system in order to identify patients at increased nutritional risk (table 1) [1,2].

Fast-Track Patients at Nutritional Risk
Postoperative complications (30-day morbidity) were graded according to their severity, and a validated therapy-orientated complication score on a 5-point scale was used [11]. Complications are reported as the number of complications, i.e. more than one complication per patient possible.

Results are expressed as medians and range values. The $\chi^{2}$ and Mann-Whitney U tests were used where appropriate. A p value of $<0.05$ was considered to indicate statistical significance. Statistical analysis was performed using the standard software package of the Statistical Package for the Social Sciences (SPSS 14.0, Inc., Chicago, Ill., USA).

\section{Results}

Patient characteristics are shown in table 2. Seven of 36 patients (19.4\%) in the FT group and 8 of 31 patients (25.8\%) in the SC group had an increased NRS $\geq 3$ (difference not significant, $\mathrm{p}=0.569$; table 3 ).

While patients without an increased nutritional risk had statistically significantly less complications in the FT versus the SC group (3/29 vs. 8/23, p = 0.044), there was no difference between patients at nutritional risk (NRS $\geq 3$ ) with $5 / 7$ and $6 / 8$ patients, respectively, with complications. Of note, major complications, i.e. grades III and 
Table 2. Patient demographics and operation characteristics

\begin{tabular}{|c|c|c|}
\hline & $\begin{array}{l}\text { Fast track } \\
(\mathrm{n}=36)\end{array}$ & $\begin{array}{l}\text { Standard care } \\
(\mathrm{n}=31)\end{array}$ \\
\hline Age, years & $60(27-86)$ & $61(39-89)$ \\
\hline Male/female & $18 / 18$ & $17 / 14$ \\
\hline ASA score I + II/III & $27 / 9$ & $20 / 11$ \\
\hline BMI & $26(19-35)$ & $26(17-33)$ \\
\hline $\mathrm{NRS}<3 / \geq 3$ & $29 / 7$ & $23 / 8$ \\
\hline Hct pre-op, $\%$ & $37(31-49)$ & $38(28-48)$ \\
\hline Malignant/benign $^{a}$ & $29 / 7$ & $29 / 2$ \\
\hline OP: left/right ${ }^{\mathrm{b}}$ & $25 / 11$ & $19 / 12$ \\
\hline OR time, $\min ^{c}$ & $140(60-190)$ & $120(55-230)$ \\
\hline Blood loss, ml & $255(0-1,100)$ & $200(0-800)$ \\
\hline
\end{tabular}

Age and BMI are medians with ranges in parentheses. ASA = American Society of Anaesthetists; BMI = body mass index; NRS = nutritional risk score Hct $=$ hematocrit.

a Underlying disease.

${ }^{\mathrm{b}}$ Operation: left- vs. right-sided hemicolectomy.

${ }^{c}$ Operation room time.

IV [11], only occurred in patients at nutritional risk, and represented almost half of all complications in these 2 patient groups (table 3). There were two bleeding complications (FT 1, SC 1), two pulmonary embolisms (FT 1, SC 1 ), and one anastomotic leak (SC). Patients at nutritional risk had significantly more complications (11/15 vs. $11 / 52$, $\mathrm{p}<0.0001)$ and a longer median hospital stay of 12 versus 7 days ( $\mathrm{p}=0.0006)$ compared to patients with a NRS $<3$. This was mainly due to more wound infections (4/15 vs. $3 / 52, \mathrm{p}=0.040)$ and overall infectious complications $(5 / 15$ vs. $5 / 52, \mathrm{p}=0.038$ ) in patients at nutritional risk (table 3 ).

Patients in the FT group had shorter hospital stays than patients in the SC group. Increased nutritional risk was associated with prolonged hospital stay, whereby patients with a NRS $\geq 3$ in the FT group had only a slightly longer hospital stay compared to patients with a NRS $<3$ (table 3).

\section{Discussion}

This study shows for the first time that FT programs can be safely performed in patients at increased nutritional risk undergoing elective colon surgery. Although these patients reveal an increased postoperative morbidity, they still benefit from a shorter hospital stay.

In accordance to current studies, we were able to confirm that the NRS can reliably identify patients at in-
Table 3. Complications and hospital stay stratified by nutritional risk

\begin{tabular}{lll}
\hline & $\begin{array}{l}\text { Fast track } \\
(\mathrm{n}=36)\end{array}$ & $\begin{array}{l}\text { Standard care } \\
(\mathrm{n}=31)\end{array}$ \\
\hline NRS $<3$ & & \\
$\quad$ Complications, $\mathrm{n}^{*}$ & $3 / 29$ & $8 / 23$ \\
$\quad$ Major complications & $0 / 29$ & $0 / 23$ \\
$\quad$ Hospital stay, days* & $5(2-14)$ & $9(6-13)$ \\
NRS $\geq 3$ & & \\
Complications, $\mathrm{n}$ & $5 / 7$ & $6 / 8$ \\
$\quad$ Major complications & $2 / 7$ & $3 / 8$ \\
Hospital stay, days & $7(5-30)$ & $14.5(8-30)$ \\
\hline
\end{tabular}

Hospital stay is given as the median with ranges in parentheses. NRS = Nutritional risk score. Major complications are defined as $\geq 3 b$ according to Dindo et al. [11]. * Statistical significance: $\mathrm{p}<0.05$.

creased nutritional risk. It is mandatory to routinely use the NRS in order to initiate preoperative nutritional support and to decrease postoperative morbidity in patients at risk $[1,2,4-6]$.

Patients with a NRS $\geq 3$ revealed an increased incidence of infectious complications and an increased severity of complications. However, it can be assumed that organ dysfunction could be limited with FT programs, since the patients at nutritional risk had a significantly shorter hospital stay in the FT versus SC group. A recent study in colorectal FT patients identified advanced age, high ASA class (III/IV), male gender and rectal surgery as risk factors for morbidity and prolonged hospital stay. The nutritional status assessed by the body mass had no predictive value [12]. On the contrary, Bozzetti et al. [13] identified weight loss and low serum albumin in 1,410 gastrointestinal cancer patients (528 colorectal) as independently associated with postoperative morbidity. Patients with perioperative nutritional support had lower complication rates. However, their patients were not included in FT programs and neither of the two studies evaluated the more comprehensive NRS as the screening tool recommended by the European Society for Clinical Nutrition and Metabolism.

The main limitation of the current analysis is the small sample size related to the fact that only two centers routinely used the NRS. The NRS was not defined as an obligatory item in the prospective randomized trial, since its clinical role was not yet established at the time of the study design $[2,5]$. Nevertheless, results should be considered as important preliminary findings, and a larger trial is mandatory for their confirmation. 
In conclusion, patients at nutritional risk (NRS $\geq 3$ ) undergoing colonic surgery must not be excluded from FT program. Careful preoperative assessment of the nutritional risk is needed to identify and treat patients with an increased nutritional risk in order to decrease postoperative complications.

\section{Acknowledgements}

We thank the members of the Zurich Fast Track Trial Group for contributing to the organization and data collection of the main study: Marco P. Zalunardo, Institute of Anesthesiology, University Hospital Zurich; Marco Decurtins, Department of
Surgery, Kantonsspital Winterthur; Lukas Eisner, Department of Surgery, Kantonsspital Olten; Italo Castelli, Department of Anesthesiology, Kantonsspital Olten; Hans P. Keller, Department of Anesthesiology, Kantonsspital Winterthur; Hans Gelpke and Felix Grieder, Department of Surgery, Kantonsspital Winterthur; Thorsten Carstensen and Andreas Gehrz, Department of Surgery, Kantonsspital Olten; Thierry Spahr, Institute of Anesthesiology, University Hospital Zurich, and Reto Paganoni, Department of Anesthesiology, Kantonsspital Olten.

\section{Conflict of Interests}

The authors received research support from Fresenius-Kabi, Switzerland, in the context of the main study.

\section{References}

- Kondrup J, Allison SP, Elia M, Vellas B, Plauth M: ESPEN guidelines for nutrition screening 2002. Clin Nutr 2003;22:415-421.

$\checkmark 2$ Kondrup J, Rasmussen HH, Hamberg O, Stanga Z: Nutritional risk screening (NRS 2002): a new method based on an analysis of controlled clinical trials. Clin Nutr 2003;22: 321-336.

3 Rasmussen HH, Kondrup J, Staun M, Ladefoged $\mathrm{K}$, Kristensen $\mathrm{H}$, Wengler A: Prevalence of patients at nutritional risk in Danish hospitals. Clin Nutr 2004;23:1009-1015.

-4 Schiesser M, Muller S, Kirchhoff P, Breitenstein S, Schafer M, Clavien PA: Assessment of a novel screening score for nutritional risk in predicting complications in gastro-intestinal surgery. Clin Nutr 2008;27:565-570.

5 Sorensen J, Kondrup J, Prokopowicz J, Schiesser M, Krahenbuhl L, Meier R, Liberda M: EuroOOPS: an international, multicentre study to implement nutritional risk screening and evaluate clinical outcome. Clin Nutr 2008;27:340-349.
6 Schwegler I, von Holzen A, Gutzwiller JP, Schlumpf R, Muhlebach S, Stanga Z: Nutritional risk is a clinical predictor of postoperative mortality and morbidity in surgery for colorectal cancer. Br J Surg 2010;97:9297.

7 Weimann A, Braga M, Harsanyi L, Laviano A, Ljungqvist O, Soeters P, Jauch KW, Kemen M, Hiesmayr JM, Horbach T, Kuse ER, Vestweber KH: ESPEN guidelines on enteral nutrition: surgery including organ transplantation. Clin Nutr 2006;25:224-244.

8 Muller S, Zalunardo MP, Hubner M, Clavien PA, Demartines N: A fast-track program reduces complications and length of hospital stay after open colonic surgery. Gastroenterology 2009;136:842-847.

9 Lassen K, Soop M, Nygren J, Cox PB, Hendry PO, Spies C, von Meyenfeldt MF, Fearon KC, Revhaug A, Norderval S, Ljungqvist O, Lobo $\mathrm{DN}$, Dejong CH: Consensus review of optimal perioperative care in colorectal surgery: Enhanced Recovery After Surgery (ERAS) Group recommendations. Arch Surg 2009; 144:961-969.
10 Wind J, Polle SW, Fung Kon Jin PH, Dejong $\mathrm{CH}$, von Meyenfeldt MF, Ubbink DT, Gouma DJ, Bemelman WA: Systematic review of enhanced recovery programmes in colonic surgery. Br J Surg 2006;93:800-809.

11 Dindo D, Demartines N, Clavien PA: Classification of surgical complications: a new proposal with evaluation in a cohort of 6336 patients and results of a survey. Ann Surg 2004;240:205-213.

- 12 Hendry PO, Hausel J, Nygren J, Lassen K, Dejong CH, Ljungqvist O, Fearon KC: Determinants of outcome after colorectal resection within an enhanced recovery programme. Br J Surg 2009;96:197-205.

13 Bozzetti F, Gianotti L, Braga M, Di Carlo V, Mariani L: Postoperative complications in gastrointestinal cancer patients: the joint role of the nutritional status and the nutritional support. Clin Nutr 2007;26:698-709. 